\title{
The preparation, characterization and application of glycol aqueous base graphene oxide nanofluid
}

\author{
Li Yihuai ${ }^{1,2,3}$, Wu Zihua ${ }^{1,3, a}$, Xie Huaqing ${ }^{1,3}$, Tang Dingcong1 ${ }^{1}$, Wang Yuanyuan ${ }^{1,3}$, Li Zhen² \\ ${ }^{1}$ Research Center of Resource Recycling Science and Engineering, Shanghai Polytechnic University, Shanghai, 201209, China \\ ${ }^{2}$ School of Environmental and Chemical Engineering, Shanghai University, Shanghai 200444, China \\ ${ }^{3}$ School of Environmental and Materials Engineering, College of Engineering, Shanghai Polytechnic University, Shanghai 201209, China
}

\begin{abstract}
Enhancement of the heat transfer of the cold side is one of the approaches to improve the performance of thermoelectric generator systems (TEGs). In order to investigate viability and further performance of the TEG for waste heat recovery in industry area, a small low-temperature waste heat thermoelectric generator setup has been constructed with graphene oxide (GO) nanofluids as coolants in the paper. The results showed excellent stability of GO nanofluids through the preparation of two-step method to be applied in the TEGs . The highest open output voltage of TEG system were obtained by $0.15 \%$ weight fraction of GO nanofluids as coolant when the temperature difference $(\triangle T)$ was designed at $95 \mathrm{~K}$ and the hot side temperature was fixed at $373 \mathrm{~K}$ in the TEGs. Compared with conventional glycol-water base fluid as coolant, the highest open voltage enhancement ratio has reached $65.26 \%$ in the TEGs.
\end{abstract}

\section{Introduction}

Thermoelectric generator (TEG) is a type of solid-state devices that can directly convert thermal energy into electricity by the Seebeck effect of semiconductor materials. Although its efficiency is a little low $(<7 \%)$, it has the noteworthy advantages of simple structures, the absence of moving parts, silence, reliability, environmental friendliness, and so on. Nowadays, TEGs have been used in many fields such as automotive waste heat, solar panel, and geothermal energy power generation, etc[1-6].

As with any heat engine operating on a thermodynamic cycle, the system of a TEG includes heat source, power system, cooling system and data acquisition system. The efficiency of the TEGs partly depends on the temperature difference between the hot and cold sides of the TEGs, which is determined by the hot source and cooling method. Usually, heat source of the TEGs are from waste heats, such as automobile exhaust, industrial waste heat, and so on[7-10]. In[11] investigated the performance of TEG heated by exhausted gas from an actual engine. Madan[12] chose dispenser printer as hot source and designed TEG devices. The conditions of the hot side of the TEG cannot change in the practical application as it is expected. So, when the temperature of the heat source was limited, the thermal transfer capabilities of the working fluid on the cold side of the TEGs directly have an effect on the output performance of the TEGs. It is a better way to improve the heat transfer at the cold side of TEG for maximizing thermal efficiency as high as possible. Until now, cooling system commonly adopts the air natural cooling or takes off residual heat by base fluids, such as water, ethylene glycol and so on. These fluids are inexpensive, availability and high specific heat capacity. However, these conventional common fluids have very low thermal conductivities[13] which limit their further development.

Nanofluid was found to exhibit better thermo-physical properties, such as higher thermal conductivity and higher viscosity[14-19].These are important factors because thermal conductivity and viscosity would directly contribute to high output performance of the TEGs. In order to improve the thermal conductivity of these conventional liquids, many researchers have focus on this aspect. Deasy[20] simulated the design of a passive liquid cooling system for TEGs and experimentally verified the simulation results. Nguven[21] experimentally studied the behavior and heat transfer enhancement of $\mathrm{Al} 2 \mathrm{O} 3$ nanofluids for cooling of microprocessors or other electronic components. Zhou[22]

a Corresponding author: yhli@sspu.edu.cn 
utilized water loaded with $\mathrm{CuO}$ nanoparticles to optimize based heat exchange schemes. The results showed the conversion efficiency of $\mathrm{CuO}$ nanofluid-cooled TEGs was $14 \%$ higher than that of pure water-cooled.

The preparation technology and performance of nanofluids have become more and more comprehensive and thorough in recent years. Graphene, found by Novoselov and co-workers[23], is a flat monolayer of sp2-bonded carbon atoms packed into a honeycomb lattice. It has attracted much attention due to twodimensional structure, unique physical and chemical properties such as high thermal conductivity[24]. It is reported that a single layer of graphene have a thermal conductivity of $5300 \mathrm{~W} / \mathrm{m} \cdot \mathrm{K}$ by experimental measurements[25]. Chen[26] experimentally obtained that a maximum thermal conductivity of suspended single-layer graphene was $2450.55 \mathrm{~W} / \mathrm{m} \cdot \mathrm{K}$. Besides, graphene is an ultralight material because of its low density which is to the benefit of nanofluids preparation. However, it is found that graphene is difficult to be dispersed in polar solvents directly due to the hydrophobic property of graphene in the process of the experiment. $\mathrm{Yu}$ [27] for the first time selected the hydrophilic graphene oxide as the additive in a nanofluid and the results showed that the thermal conductivity enhancement ratios of GO nanosheets nanofluids were up to $76.80 \%$ compared to the corresponding base fluid. The team further developed a facile technique to produce ethylene glycol based nanofluids containing GO nanosheets and the thermal conductivity enhancement ratios were up to 86\% [24]. Ijam[28] prepared GO nanosheets /glycol-water-based nanofluids which kept stable for more than 5 months, the results showed that the improvement in thermal conductivity was up to $11.7 \%$. And then, hydrophilic graphene nanofluids have been explored for practical applications instead of traditional fluids, such as in solar collectors[29-31], miniature loop heat pipe[32-35], and so on. It is proved that GO and homologous series nanofluids are effective media of heat transfer. In the study, GO and its stable nanofluids were prepared by the chemical and two-step method, respectively. An experimental scale test system of TEG was constructed and GO nanofluids as coolant were used instead of base fluid in the cooling system. The effect of GO nanofluids on the output open voltage of the TEG system at different concentration and different temperature were studied. The results could provide a fundamental understanding of the optimization of largescale TEG systems.

\section{Experimental}

Graphite (code no.GF-1S2) was from by Shanghai Shanshan Technology limited Company. Other chemical reagents $\left(\mathrm{H}_{2} \mathrm{SO}_{4}, \mathrm{H}_{3} \mathrm{PO}_{4}, \mathrm{KMnO}_{4}, \mathrm{H}_{2} \mathrm{O}_{2}\right)$ were analytical reagent and from Sinopharm Chemical Reagent Co. Ltd.. All reagents were used without any further treatment. The synthesis of GO was adopted from Huang et al.[36] based on modified Hummer's method.

The prepared GO were dispersed in the mixture with ethylene glycol and deionized water $(\mathrm{V} / \mathrm{V}=1: 1)$ without adding any surfactant solution. The mixture is homogenized by ultrasonication (KQ2200E Sonicator, KunShan Ultrasonic Instruments Co.Ltd., China) for about 1 hour to suspend the GO in mixed reagent. A larger fraction of $0.5 \mathrm{wt} . \%$ GO nanofluid was prepared according to the above two-step process and it was diluted to prepare $0.05 \mathrm{wt} . \%, 0.10 \mathrm{wt} . \%, 0.15 \mathrm{wt} . \%$ and $0.20 \mathrm{wt} . \%$ GO nanofluids by severely magnetic stirring and ultrasonication. X-ray diffraction (XRD) patterns of the selected samples were recorded by the reflection scan with nickel-filtered $\mathrm{Cu} \mathrm{K} \alpha$ radiation (D8, BrukerAXS, Germany); The Raman spectra were gained with a FT-Raman microscope using a laser with a wavelength of 532nm (Senterra, Bruker, Germany); The thermal conductivity of samples was measured with a thermal conductivity analyzer (C-Therm TCi, C-Therm Technologies Ltd., Canada); Viscosity of GO nanofluids was measured as a function of temperatures using viscometers (DV2THA, Brookfield, USA).

The TEG test system and measurement uncertainties were described in detail in our previous paper [37]. The schematic of the experimental system is shown in Fig.1. The system was made up of a pump (Masterflex L/P, 77962-20, USA), a thermostatic bath (DCW-2008, Ning Bo Scientz Biotechnology Co., Ltd., China), TEG, a heating plate (IKA, C-MAG-HP4, Germany), a heat sink and a data acquisition unit. The interfaces between the TEG, a heating plate and a heat sink were covered thermal grease to reduce the thermal contact resistance. The detailed parameters of TEG are shown in table 1. GO nanofluids as coolant were poured into the liquid cabin firstly and then were brought into the whole TEG system by pump. The open output voltage $(\mathrm{V})$ and the cold surface temperature of TEG were collected by the data acquisition system, for example, the open output voltage of TEG was detected by the electrical leads line 2 and 3 , the inlet temperature $\left(\mathrm{T}_{\mathrm{in}}\right)$, the outlet temperature ( $T_{\text {out }}$ ) and the designed condenser's temperature $\left(\mathrm{T}_{\mathrm{w}}\right)$ were obtained by the thermocouples line 4, 5 and 1 , respectively. When the temperature at the hot side of TEGs is fixed, the voltage of TEGs depended on the performance of the cold side of TEGs. All the experimental data were got by repeated more than twice. 


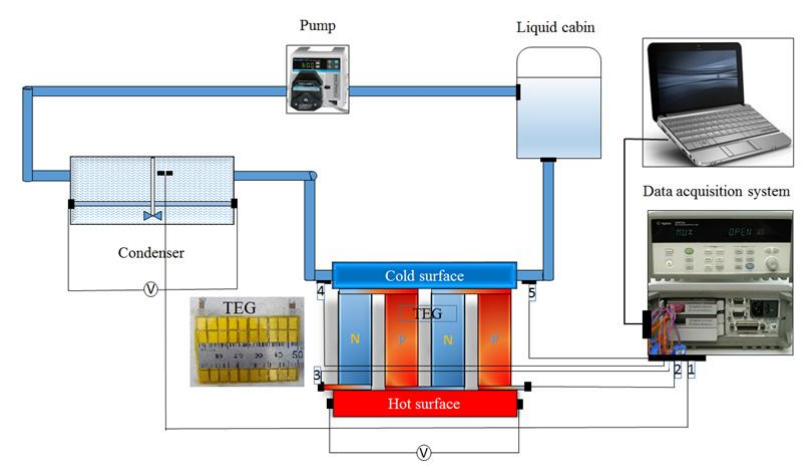

Fig.1 Schematic of the experimental setup

Table 1 Specifications of the TEG

\begin{tabular}{|c|c|}
\hline Specifications & Value \\
\hline Type of material & $\mathrm{Bi}_{2} \mathrm{Te}_{3}$ \\
\hline Maximum current $(\mathrm{A})$ & 7 \\
\hline Maximum voltage $(\mathrm{V})$ & 3.5 \\
\hline Maximum power $(\mathrm{W})$ & 7.6 \\
\hline$\triangle \mathrm{Tmax}\left({ }^{\circ} \mathrm{C}\right)$ & 270 \\
\hline Dimensions $(\mathrm{L} \times \mathrm{W} \times \mathrm{H})\left(\mathrm{mm}^{3}\right)$ & $50 * 50 * 3.5$ \\
\hline Number of couples & 49 \\
\hline
\end{tabular}

\section{Results and discussions}

\subsection{Micro-structure characterization}

Fig.2 shows the XRD results of graphene oxide and the pristine graphite. The sharp diffraction peak observed at $2 \theta=26.501^{\circ}$ associated with (002) reflection of graphite and interlayer distance of $0.336 \mathrm{~nm}$ calculated by Bragg' s equation. After oxidation of graphite, a strong peak at $11.46 \mathrm{o}$ associated with (001) reflection of $\mathrm{GO}$ with an interlayer distance of $0.77 \mathrm{~nm}$, the stacking height and the number of layers are $5 \mathrm{~nm}$ and about 7, respectively, calculated by Scherrer equation. The increase in interlayer distance is due to the included functional groups between the graphene sheets after the oxidation[28] .

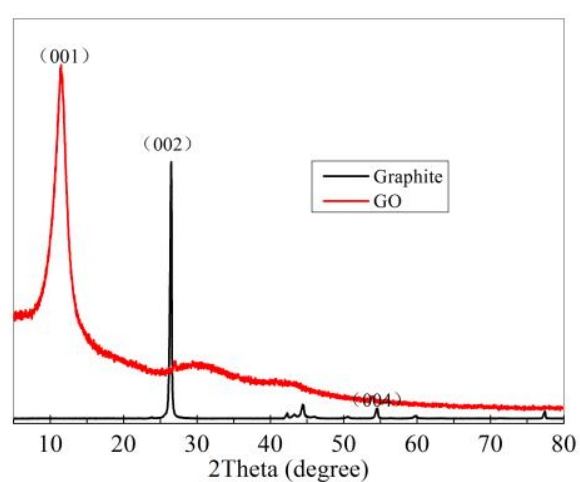

Fig.2 XRD patterns for graphite and GO

Raman spectroscopy is a widely used tool for the characterization of the structure of graphitic materials. From Fig.3, the main feature in Raman spectra of graphitic materials are so-called $\mathrm{G}$ and $\mathrm{D}$ peaks, which lie at around 1560 and $1360 \mathrm{~cm}-1$ for visible excitation[38]. The $\mathrm{G}$ peak is caused by the Raman active E2g phonon of $\mathrm{C}$ sp2 atoms. The D peak refers to the sp3 hybridization of carbon atoms and structure defect and its relative signal strength (compared to the $G$ peak) depends strongly on the amount of disorder in the carbon material[39]. In graphite, the ratio of D peak to $G$ peak intensity (ID/IG) is 0.28 indicating that the graphite is ordered structure with low defects; In graphene oxide, the G peaks is shifted and broadened to $1590 \mathrm{~cm}-1$ and the D peaks is shifted to $1540 \mathrm{~cm}-1$, the ratio of ID/IG is 1.04 which is higher than that of graphite. This is due to severe oxidation of graphite by strong acid during preparation and the more defects caused. Besides, since this ratio is greater than 1 , it is plausible to say that some oxygen groups on the surface of graphene were introduced during the preparation[40]. As described in the reference[41], there is no doubt that the high thermal conductivity is diminished by defects and this defects have direct influence on the heat transport, but this defects are very beneficial for the stability of the GO nanofluids without surfactant addition.

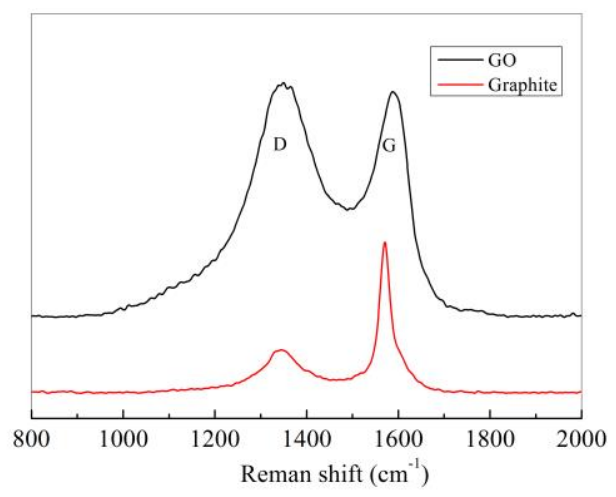

Fig.3 Raman spectra for graphite and GO. 


\subsection{Effect of GO nanofluids on the voltage of the TEG}

The effect of GO nanofluids on the heat exchange of cold side of the TEGs was studied. Fig.4 shows the distribution of $\mathrm{T}_{\text {ave }}\left(\mathrm{T}_{\text {ave }}=\left(\mathrm{T}_{\text {in }}+\mathrm{T}_{\text {out }}\right) / 2\right)$ and $\mathrm{T}_{\mathrm{w}}$ versus time. The results indicated that the temperature curves were nearly a straight line when time was over 900s and reaches a steady state of the system. All the experimental data were collected when the starting time of system was over 900s. Besides, it was found that the fluctuation of the temperatures with $0.15 \mathrm{wt} . \% \mathrm{GO}$ nanofluid as the coolant are larger than those of the base fluid as the coolant. The result may be caused by Brownian movement of nanoparticles dispersed in the base fluid.

Fig.5 presents the effect of the GO nanofluids on the voltage of the TEG at different temperature and flow rate. The hot side temperature was fixed at $373 \mathrm{~K}$, The designed temperature difference $(\triangle T)$ was obtained through varying the cold side fluid temperature. The highest voltage were 0.432 and $0.412 \mathrm{~V}$ with $0.15 \mathrm{wt} . \%$ GO nanofluids as coolant when $\triangle \mathrm{T}$ was at 95 and $90 \mathrm{~K}$, respectively. The voltage improvement ratio is up to $4.85 \%$ when $\triangle \mathrm{T}$ was increased by $5 \mathrm{~K}$ at the test system of the TEG. The change of voltage of the TEG at different concentration and same temperature difference can be observed from Fig.5 (a). When $\triangle$ $\mathrm{T}=95 \mathrm{~K}$, the voltage of the TEG increases with increasing the particles fraction when it is lower than $0.15 \mathrm{wt} . \%$. While the fraction is higher than $0.15 \mathrm{wt} . \%$, the voltage of the TEG decrease with increasing solid loading. The same tendency occurred at $90 \mathrm{~K}$ which can be seen from Fig.5 (b). The enhancement percentage was calculated based on the expression below:

Enhancement ratio $=\left(\left(\mathrm{V}-\mathrm{V}_{0}\right) / \mathrm{V}_{0}\right) \times 100 \%$

where $\mathrm{V}$ and Vo are the open voltage of the tested TEG device with nanofluid and the base fluid as the coolant, respectively. The highest enhancement of $60 \%, 65 \%$ were achieved at $\triangle \mathrm{T}=90$ and $95 \mathrm{~K}$ with $0.15 \mathrm{wt} . \% \mathrm{GO}$ nanofluid rather than 0.20 wt.\% GO nanofluid, respectively. This behavior may be due to more GO added in the base liquid would increase the thermal conductivity as well as the viscosity
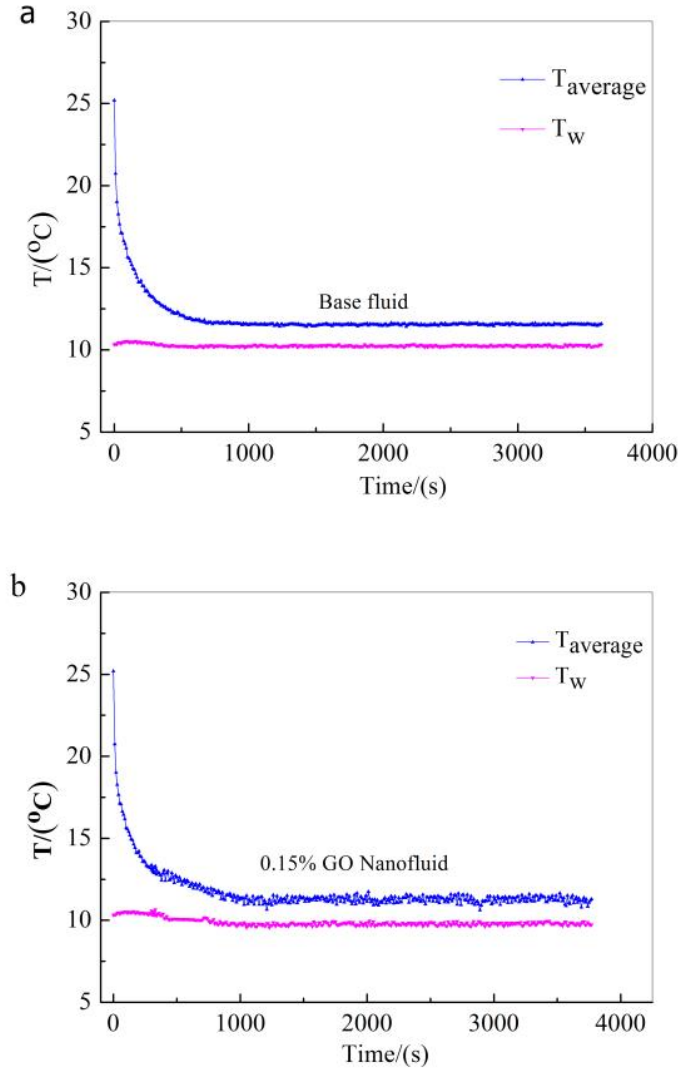

Fig.4 Temperature distributions of $\mathrm{T}_{\text {average }}$ and $\mathrm{T}_{\mathrm{w}}$ with base fluid and $0.15 \mathrm{wt} . \% \mathrm{GO}$ nanofluids
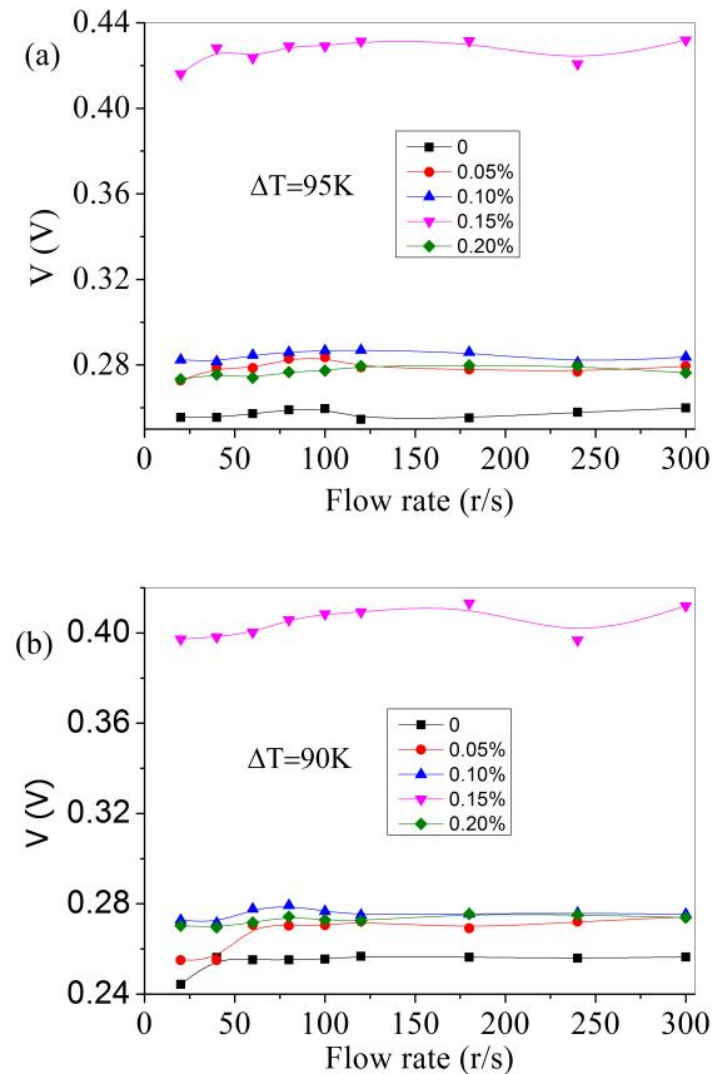
Fig.5 Effect of the GO nanofluid on the voltage of TEGs at different conditions

\section{Conclusions}

In the present experimental study, GO have been prepared by using Modified Hummer's method and the GO dispersing glycol-water based nanofluids were further prepared by a two-step method. The interlayer spacing of GO increases due to the formation of functional groups between the graphite layers and more defects in the structure of GO after oxidation. These functional groups and defects are the main reasons of the stability of GO nanofluids without any surfactant addition. To thoroughly investigate the heat transfer enhancement and behavior of GO nanofluid as coolant of the TEGs. The open output voltage of TEG can be increased nearly $60 \%$ and $65 \%$ compared with glycol-water mixed base fluid at the weight fraction of $0.15 \%$ when the temperature differences $(\triangle \mathrm{T})$ are 90 and $95 \mathrm{~K}$, respectively. So, in a word, using GO nanofluids is an effective approach to enhance the heat transfer performance of the coolant in the TEGs.

\section{Acknowledgements}

This work was supported by the National Natural Science Foundation of China (Grant Nos. 51676117\& 51476095), the Natural Science Foundation of Shanghai (Grant No. 14ZR1417000), the Program for Professor of Special Appointment (Young Eastern Scholar, Grant No. QD2015052) at Shanghai Institutions of Higher Learning, the Key Subject of Shanghai Polytechnic University (Material Science and Engineering, Grant No.XXKZD1601) and Gaoyuan Discipline of Shanghai - Environmental Science and Engineering (Resource Recycling Science and Engineering).

\section{References}

1. T. Wang, W. Luan, W. Wang, S.-T. Tu, Applied Energy, 136 (2014) 860-865.

2. W. He, S. Wang, X. Zhang, Y. Li, C. Lu, Energy, 91 (2015) 1-9.

3. S. Islam, I. Dincer, B.S. Yilbas, Energy, 93, Part 1 (2015) 1246-1258.

4. M.F. Remeli, L. Kiatbodin, B. Singh, K. Verojporn, A. Date, A. Akbarzadeh, Energy Procedia, 75 (2015) 645-650.

5. T.H. Kwan, X. Wu, Applied Energy, 165 (2016) 297-307.

6. G. Li, G. Zhang, W. He, J. Ji, S. Lv, X. Chen, H. Chen, Energy Conversion and Management, 112 (2016) 191-198.
7. L. Wang, W. Cai, H. Zhao, C. Lin, J. Yan, Applied Thermal Engineering, 94 (2016) 314-323.

8. İ. Temizer, C. İlkılıç, Renewable and Sustainable Energy Reviews, 63 (2016) 141-151.

9. J.-H. Meng, X.-D. Wang, W.-H. Chen, Energy Conversion and Management, 120 (2016) 71-80.

10. X. Wang, B. Li, Y. Yan, S. Liu, J. Li, Applied Thermal Engineering, 102 (2016) 176-183.

11. B.d. In, H.i. Kim, J.w. Son, K.h. Lee, International Journal of Heat and Mass Transfer, 86 (2015) 667680.

12. D. Madan, Z. Wang, P.K. Wright, J.W. Evans, Applied Energy, 156 (2015) 587-592.

13. M. Afrand, A.A. Nadooshan, M. Hassani, H. Yarmand, M. Dahari, International Communications in Heat and Mass Transfer, 77 (2016) 49-53.

14. C. Selvam, E.C. Muhammed Irshad, D.M. Lal, S. Harish, Experimental Thermal and Fluid Science, 77 (2016) 188-196.

15. A.M. Aly, S.E. Ahmed, Journal of Nanofluids, 5 (2016) 110-119.

16. M.A. Akhavan-Behabadi, F. Hekmatipour, B. Sajadi, Experimental Thermal and Fluid Science, 78 (2016) 10-17.

17. M.A. Akhavan-Behabadi, M. Shahidi, M.R. Aligoodarz, M. Fakoor-Pakdaman, Applied Thermal Engineering, 106 (2016) 916-924.

18. Q. Li, A. Shirazi, C. Zheng, G. Rosengarten, J.A Scott, R.A. Taylor, Energy, 96 (2016) 253-267.

19. M. Hosseinzadeh, S.Z. Heris, A. Beheshti, M. Shanbedi, Journal of Thermal Analysis and Calorimetry, 124 (2016) 827-838.

20. M.J. Deasy, N. Baudin, S.M. O'Shaughnessy, A.J. Robinson, Applied Energy, 205 (2017) 499-510.

21. C.T. Nguyen, G. Roy, C. Gauthier, N. Galanis, Applied Thermal Engineering, 27 (2007) 1501-1506.

22. S. Zhou, B.G. Sammakia, B. White, P. Borgesen, C. Chen, International Journal of Heat and Mass Transfer, 81 (2015) 639-645.

23. A.K. Geim, K.S. Novoselov, Nat Mater, 6 (2007) 183-191.

24. W. Yu, H. Xie, X. Wang, X. Wang, Physics Letters A, 375 (2011) 1323-1328.

25. A.A. Balandin, S. Ghosh, W. Bao, I. Calizo, D. Teweldebrhan, F. Miao, C.N. Lau, Nano Letters, 8 (2008) 902-907.

26. L. Chen, H. Xie, W. Yu, B. Wang, Z. Wu, International Journal of Thermal Sciences, 94 (2015) 221-227.

27. W. Yu, H. Xie, W. Chen, Journal of Applied Physics, 107 (2010).

28. A. Ijam, A. Moradi Golsheikh, R. Saidur, P. Ganesan, Journal of Materials Science, 49 (2014) 5934-5944.

29. M. Vakili, S.M. Hosseinalipour, S. Delfani, S. Khosrojerdi, Solar Energy Materials and Solar Cells, 152 (2016) 187-191.

30. S. Mesgari, R.A. Taylor, N.E. Hjerrild, F. Crisostomo, Q. Li, J. Scott, Solar Energy Materials and Solar Cells, 157 (2016) 652-659.

31. A.K. Hussein, Renewable and Sustainable Energy Reviews, 62 (2016) 767-792. 
32. T. Tharayil, L.G. Asirvatham, V. Ravindran, S. Wongwises, International Journal of Heat and Mass Transfer, 93 (2016) 957-968.

33. E. Sadeghinezhad, M. Mehrali, M.A. Rosen, A.R. Akhiani, S. Tahan Latibari, M. Mehrali, H.S.C. Metselaar, Applied Thermal Engineering, 100 (2016) 775-787.

34. M. Mehrali, E. Sadeghinezhad, R. Azizian, A.R. Akhiani, S.T. Latibari, M. Mehrali, H.S.C. Metselaar, Energy Conversion and Management, 118 (2016) 459-473.

35. K.M. Kim, I.C. Bang, International Journal of Thermal Sciences, 100 (2016) 346-356.

36. N.M. Huang, H.N. Lim, C.H. Chia, M.A. Yarmo, M.R. Muhamad, International journal of nanomedicine, 6 (2011) 3443-3448.

37. Y. Li, Z. Wu, H. Xie, J. Xing, J. Mao, Y. Wang, Z. Li, Science China Technological Sciences, 60 (2017) 1168-1174.

38. K.N. Kudin, B. Ozbas, H.C. Schniepp, R.K. Prud'homme, I.A. Aksay, R. Car, Nano Letters, 8 (2008) 36-41.

39. W.S. Sarsam, A. Amiri, M.N.M. Zubir, H. Yarmand, S.N. Kazi, A. Badarudin, Colloids and Surfaces aPhysicochemical and Engineering Aspects, 500 (2016) 17-31.

40. M.B. Moghaddam, E.K. Goharshadi, M.H. Entezari, P. Nancarrow, Chemical Engineering Journal, 231 (2013) 365-372.

41. H. Xie, W. Yu, Y. Li, L. Chen, Nanoscale Research Letters, 6 (2011) 124. 